Bio - grafía. Escritos sobre la Biología y su Enseñanza. ISSN 2027-1034

Edición Extraordinaria. p.p. 1238 - 1246

Memorias del IX Encuentro Nacional de Experiencias en Enseñanza de la Biología y la Educación Ambiental. IV Congreso Nacional de Investigación en Enseñanza de la Biología.

\title{
EL APRENDIZAJE POR INDAGACIÓN HACIA LA ALFABETIZACIÓN CIENTÍFICA DE LOS ESTUDIANTE EN LAIE NO. 3 SANT A CATALINA DE SIENA DE MAICAO - LA GUAJIRA
}

\author{
Carlos Milciades Mendoza Vergara ${ }^{1}$ \\ Carlos Humberto Barreto Tovar ${ }^{2}$
}

\section{RESUMEN}

La indagación a nivel escolar es una estrategia de enseñanza y aprendizaje para el desarrollo de las competencias y el pensamiento científico de los estudiantes, así como el fortalecimiento de los procesos de enseñanza del docente en su alfabetización académica. En este trabajo se evidencian los diferentes niveles de observación e indagación que tienen los estudiantes, así como las reflexiones realizada por el docente investigador de sus conocimientos profesionales evidenciados en los niveles de desempeño del pensamiento científico de los estudiantes. Se convierte en una propuesta pedagógica que lleva a la formación de los futuros ciudadanos alfabetizados científicamente para que tomen decisiones responsables frente a la ciencia y la tecnología en su contexto cotidiano.

PALABRAS CLAVES: Indagación, alfabetización académica, alfabetización, pensamiento científico, competencias, conocimiento profesional del profesor de ciencias.

\section{ABSTRACT}

The inquiry at the school level is a teaching and learning strategy for the development of students' competences and scientific thinking, as well as the strengthening of teacher education processes based on their academic literacy. In this paper, the students 'different levels of observation and inquiry are evidenced, as well as the reflections made by the researcher of his / her professional knowledge evidenced in the performance levels of students' scientific thinking. It becomes a pedagogical proposal that leads to the training of future citizens scientifically literate so that they make responsible decisions regarding science and technology in their daily context.

KEYWORDS: Inquiry, academic literacy, literacy, scientific thinking, skills, reflection, professional knowledge of the science teacher

\footnotetext{
${ }^{1}$ Universidad de La Sabana. Facultad de Educación. Maestría en Pedagogía Ext. La Guajira. Correo: carlosmeve@unisabana.edu.co

2 Universidad de La Sabana. Facultad de Educación. Maestría en Pedagogía Ext. La Guajira. Correo: carlos.barreto2@unisabana.edu.co
} 


\author{
Bio - grafía. Escritos sobre la Biología y su Enseñanza. ISSN 2027-1034 \\ Edición Extraordinaria. p.p. 1238 - 1246 \\ Memorias del IX Encuentro Nacional de Experiencias en Enseñanza de la Biología y la \\ Educación Ambiental. IV Congreso Nacional de Investigación en Enseñanza de la \\ Biología.
}

\title{
INTRODUCCIÓN
}

La enseñanza de las ciencias está orientada con base en las finalidades y objetivos definidos en los referentes conceptuales de los estándares básicos de competencias (MEN, 2006). En este sentido, el grupo de acciones concretas de pensamiento y de producción consignadas están formuladas a partir de la alfabetización científica como la capacidad de usar el conocimiento científico en la toma de decisiones (Meinardi, 2010, p.26). Sin embargo, mientras no se realicen reflexiones y evaluaciones pertinentes sobre los niveles del pensamiento científico desarrollados en las aulas de clases predominarán enfoques didácticos fundamentados en la transmisión expositiva, sin relevancia personal y social para los estudiantes. En esa línea, una perspectiva pedagógica capaz de desarrollar una verdadera alfabetización científica es el aprendizaje por indagación, ya que es una metodología que potencializa las habilidades del pensamiento crítico.

\section{Contexto}

La institución educativa No. 3 sede Santa Catalina del Municipio de Maicao está ubicado en el extremo sur de la península de La Guajira, más exactamente en la zona de transición entre la baja y la alta Guajira. La población estudiantil del colegio está conformada por 2805 estudiantes. La investigación fue realizada en el grado décimo que está conformado por 39 estudiantes; 19 niñas y 20 niños, los cuales oscilan en edades desde los 14 hasta los 18 años. Desde el punto de vista socioeconómico el $90 \%$ de la población estudiantil pertenece a los estratos 1 y 2, así mismo, la mayor parte de las familias se sustentan en actividades económicas basadas en la informalidad, muchos de ellos son comerciantes independientes y vendedores de gasolina, por otra parte, un pequeño porcentaje está representado por acudientes vinculados al trabajo formal. De igual manera, es importante considerar la diversidad cultural de la comunidad educativa representada por las etnias Wayuu, Zenú, afrodescendientes y mestizos provenientes de las distintas regiones del país. Es de resaltar que en las aulas de clases concurren ocasionalmente estudiantes árabes de origen libanés y últimamente también se registra una gran afluencia de estudiantes de nacionalidad venezolana.

\section{Problemática}

Cabe iniciar mencionando que las metodologías expositivas dificultan el desarrollo del pensamiento científico del niño, ya que, "cada vez que se le enseña prematuramente a un niño algo que hubiera podido descubrir solo, se le impide a ese niño inventarlo, y en consecuencia, entenderlo, completamente" Piaget (citado en Pozo y Carretero, 1987). Así mismo, en la transmisión expositiva del conocimiento predomina el uso de preguntas fácticas, sin tener en cuenta el contexto, ni las experiencias de los estudiantes, sino tomando como referencia las teorías presentadas en los diferentes tipos textos de fácil acceso y manejados ampliamente por el docente. Bajo esta perspectiva, no se promueve la alfabetización científica dado que se desarrolla un individuo sin capacidad para tomar 
Bio - grafía. Escritos sobre la Biología y su Enseñanza. ISSN 2027-1034

Edición Extraordinaria. p.p. 1238 - 1246

Memorias del IX Encuentro Nacional de Experiencias en Enseñanza de la Biología y la

Educación Ambiental. IV Congreso Nacional de Investigación en Enseñanza de la Biología.

decisiones y proponer alternativas de solución ante cualquier problemática o circunstancia.

Es importante tener en cuenta los resultados de las Pruebas Saber y el desempeño observado en el aula de clase para identificar las dificultades para el desarrollo del pensamiento científico de los estudiantes. En este sentido, se obtiene que el $86,54 \%$ de los estudiantes de la Institución Educativa No. 3 de Maicao no elaboran conclusiones y predicciones a partir de información derivada de investigaciones científicas, no reconocen patrones y regularidades en los datos derivados de una investigación científica, no representan datos e información de diferentes contextos, además de tener dificultades para interpretar y relacionar información presentada en tablas y distintos tipos de gráficas con conceptos de las ciencias.

\section{METODOLOGÍA}

El enfoque de la investigación es de tipo cualitativo fundamentado en la investigación acción, que según Simmons (citado por Latorre, 1992) se concibe como un proceso de indagación sistemática de conocimientos, destrezas y actitudes, que le permite al profesor de manera individual o colectiva investigar, comprender y solucionar problemas de enseñanza y aprendizaje que tienen lugar en el desarrollo de las clases. La información se recoge a partir de técnicas coherentes con el enfoque cualitativo como son los diarios de campo, grabaciones de audio y video, además de transcripciones.

En este sentido, se desarrollaron los correspondientes procesos de planificación, acción observación y reflexión. En primer lugar, se planificaron las actividades de observación e indagación a realizar por los estudiantes de grado décimo desde el marco conceptual de la Enseñanza para la Comprensión (EpC); así mismo se realizó la grabación y la posterior observación del desarrollo de la clase registrando la información en forma de guión. La reflexión se construye a partir de la relación de los niveles de observación e indagación evidenciados por los estudiantes y el análisis de los componentes del Conocimiento Profesional del Profesor de Ciencias (CPPC).

\section{RESULTADOS Y DISCUSIÓN}

La escuela debe promover actividades que lleven a los estudiantes al planteamiento de preguntas, problemas y alternativas de solución a partir de su entorno, sus intereses y sus necesidades. En tal sentido, es necesario rediseñar las estrategias de enseñanza para diagnosticar, desarrollar y fortalecer las habilidades científicas. Con esto en mente, desde el marco de enseñanza para la comprensión se planificó a partir del tópico "Frascos de laboratorio para reactivos" (figura 1) una actividad diagnóstica para valorar los niveles de observación e indagación de los estudiantes de la Institución Educativa No 3 de Maicao. Desde esta perspectiva se caracterizaron los niveles de observación e indagación de los estudiantes de décimo grado según Santelices (1989) y Maloka (2003) respectivamente. 
Bio - grafía. Escritos sobre la Biología y su Enseñanza. ISSN 2027-1034

Edición Extraordinaria. p.p. 1238 - 1246

Memorias del IX Encuentro Nacional de Experiencias en Enseñanza de la Biología y la

Educación Ambiental. IV Congreso Nacional de Investigación en Enseñanza de la Biología.

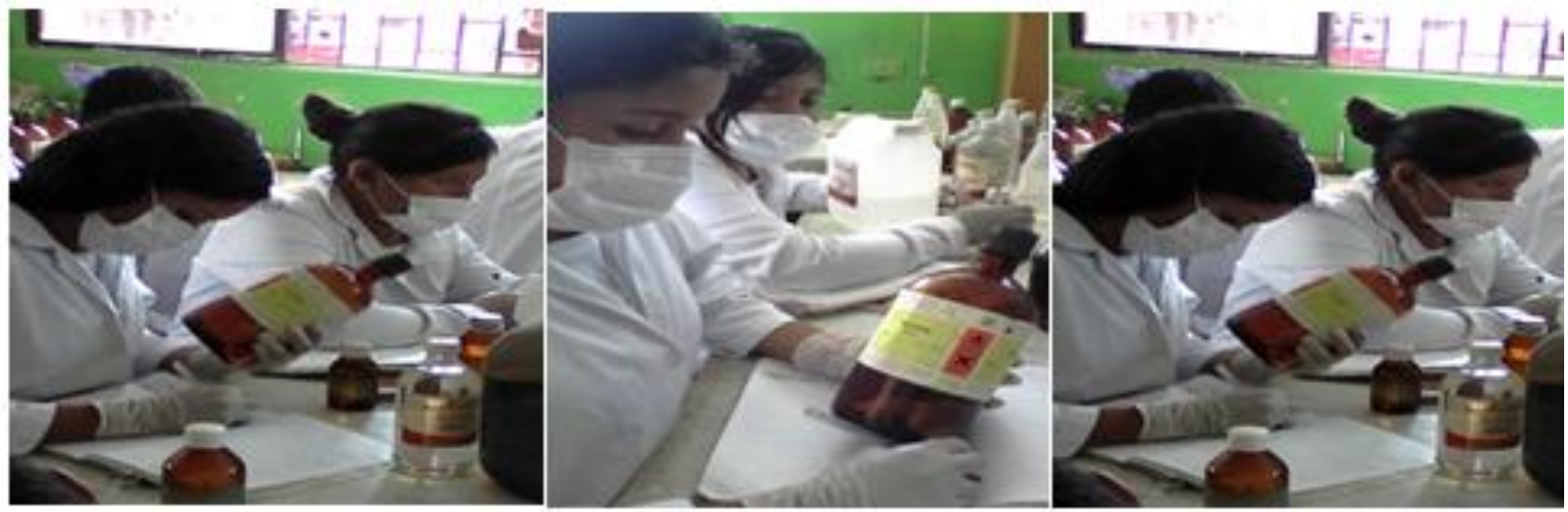

\section{Fiqura 1: Fase de observación}

Con respecto a los resultados de la actividad de indagación planeada se obtuvo que la observación en los estudiantes del grado décimo de la Institución Educativa No 3 de Maicao corresponde en un $93 \%$ ubicado al nivel 1A y un 7\% al nivel 3B (figura 2). Así mismo, se evidenció a partir de las preguntas elaboradas por los estudiantes que el $22 \%$ están orientadas a obtener un dato o concepto, el $34 \%$ buscan una causa explicativa y el $44 \%$ corresponden a preguntas atípicas (figura 3 y 4). En consecuencia, el desempeño de los estudiantes de décimo grado de esta Institución Educativa se limitó solamente a identificar o reconocer formas básicas de la información, describir cambios en los objetos y a realizar un procedimiento sin comprenderlo.

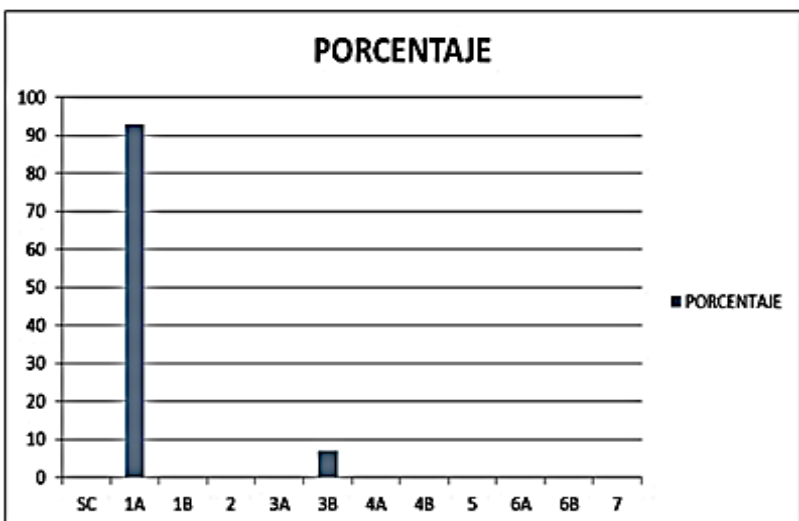

Figura 2: Nivel de observación de los estudiantes de décimo grado de la IE No 3 de Maicao según Santelices (1989)

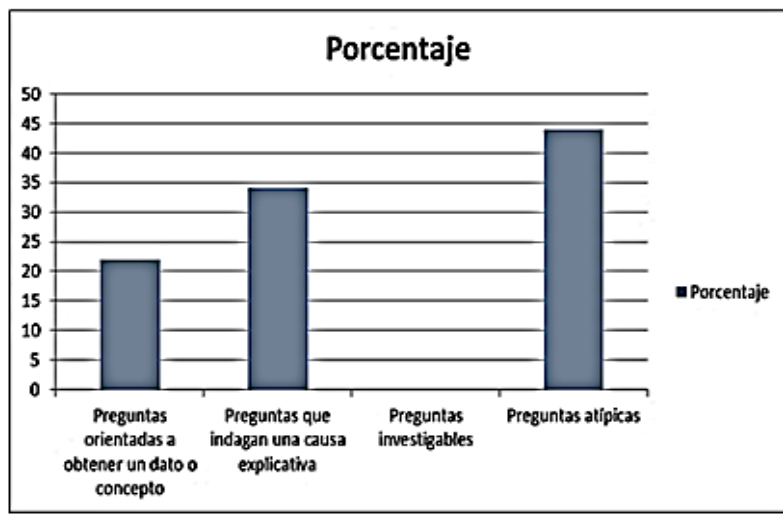

Figura 3: Nivel de indagación de los estudiantes de décimo grado de la IE No 3 de Maicao segun Maloka 2003 
Bio - grafía. Escritos sobre la Biología y su Enseñanza. ISSN 2027-1034

Edición Extraordinaria. p.p. 1238 - 1246

Memorias del IX Encuentro Nacional de Experiencias en Enseñanza de la Biología y la

Educación Ambiental. IV Congreso Nacional de Investigación en Enseñanza de la Biología.

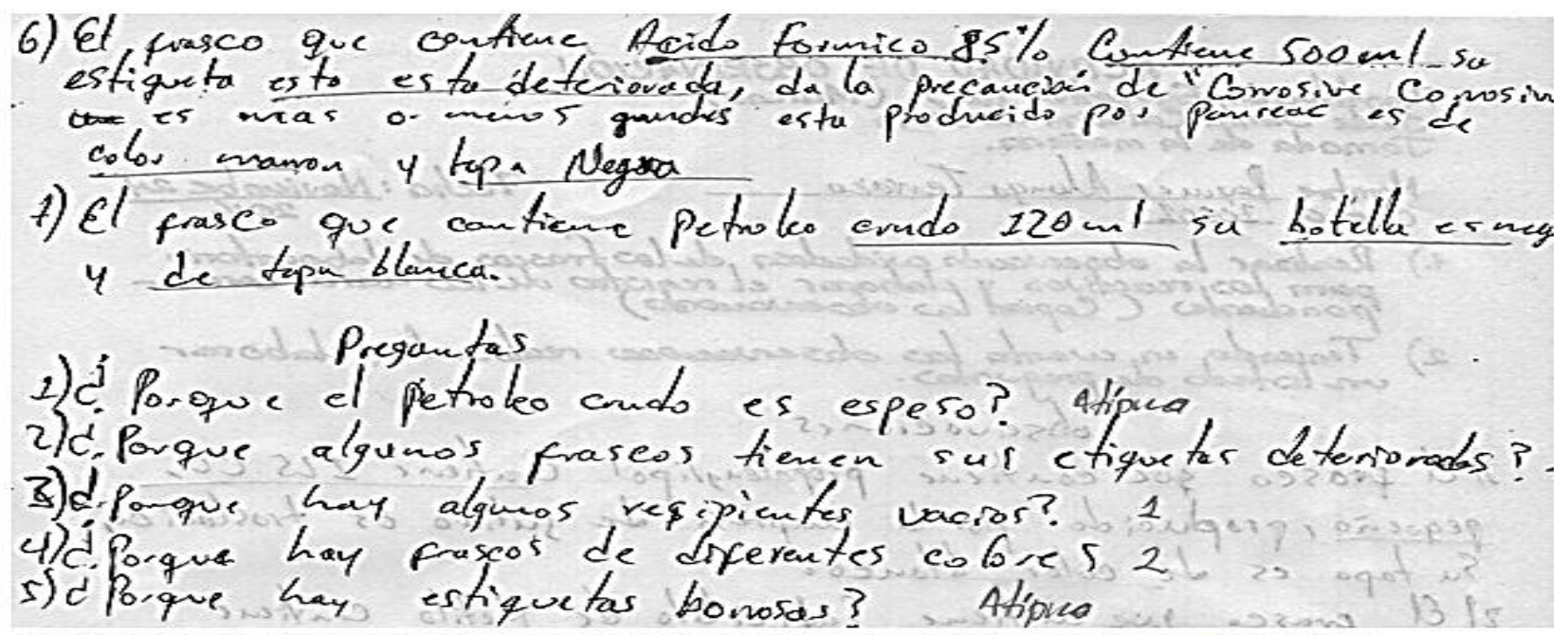

Figura 4: Observaciones y preguntas elaboradas por los estudiantes de la IE No 3 de Maicao

Para dar pasos firmes hacia el desarrollo del pensamiento científico que lleve a los estudiantes a una verdadera alfabetización científica, es conveniente que el docente se pregunte cómo influye su labor en los resultados reflejados en los estudiantes, además que a nivel institucional trabaje en equipo para indagar y fortalecer las prácticas de aula mediante propuestas pedagógicas que potencien las habilidades de pensamiento científico. En este sentido, el docente tiene la oportunidad de aprovechar la información del contexto de enseñanza y aprendizaje para reflexionar, sistematizar, evaluar y elaborar propuestas que fortalezcan el desarrollo del pensamiento científico.

Cabe resaltar que el uso de planteamientos pedagógicos alejados de la realidad del aula del docente además de no brindar soluciones contextualizadas, también contribuirán con la concepción del docente de la ruptura entre la teoría y la práctica de su labor de enseñanza. En consecuencia, la perspectiva y labor del docente puede apoyarse significativamente en los componentes de la investigación acción, como una estrategia reflexiva que resignifique su reconocimiento y su importancia en la transformación de la realidad social de las comunidades y del país en general.

Respecto a la indagación como estrategia de enseñanza se cuenta con una amplia gama de fundamentos teóricos validados por la comunidad académica, que no son de referencia reciente, por ejemplo, Bachelard (1938) señala que: "todo conocimiento es la respuesta a una pregunta" (citado en Carvalho, 1997). En este sentido, surgen interrogantes como, ¿Por qué actualmente en los procesos de enseñanza y aprendizaje se mantienen métodos transmisivos y memorísticos de la información?, ¿cómo se puede cambiar este enfoque de enseñanza?

En referencia a los requerimientos de una labor enseñanza consciente, racional y específica, es necesario, que el docente realice un análisis reflexivo de los componentes de su conocimiento profesional y su incidencia en el desarrollo del pensamiento científico de los estudiantes, reflejado en los resultados de las evaluaciones tanto a nivel institucional como en las pruebas 


\section{Bio - grafía. Escritos sobre la Biología y su Enseñanza. ISSN 2027-1034}

\section{Edición Extraordinaria. p.p. 1238 - 1246}

\section{Memorias del IX Encuentro Nacional de Experiencias en Enseñanza de la Biología y la}

Educación Ambiental. IV Congreso Nacional de Investigación en Enseñanza de la Biología.

externas. Puesto que la reflexión es la herramienta principal para cambiar la concepción tradicional de los procesos de enseñanza y aprendizaje, que se manifiestan como la adquisición de una competencia, habilidad o capacidad, que en función de su alcance permite juzgar o valorar al aprendiz según mencionan Wilson y Meyers (citados por Díaz, 2006). Por el contrario, las valoraciones del aprendizaje de los estudiantes deben percibirse como un insumo para que el docente juzgue y valore la pertinencia de sus prácticas de aula. En este sentido, "el docente se convierte a la vez en un estudiante de su propia forma de enseñar, es decir, en alguien que indaga y reflexiona sobre su propia práctica como enseñante" (Díaz, 2006, p. 15).

En lo que se refiere a esta forma de valorar los procesos de enseñanza y aprendizaje, se cuenta con una gran variedad de acciones e instrumentos que la hacen posible. En primer lugar, el docente debe apoyarse en una de las habilidades básicas de la indagación, tal como es, la observación. En relación con esta habilidad, Porlán, Rivero y Martín del Pozo (1997, p.158) plantean "que observarse a uno mismo, o compartir los datos de la observación de otro, significa con frecuencia el descubrimiento de ciertas pautas de acción que desconocíamos". En la medida en que el profesor realice una observación reflexiva de las prácticas de aula, tomando como referencia el análisis de las planeaciones de clases y los componentes del conocimiento profesional de profesor, tiene la oportunidad de identificar las múltiples representaciones, rutinas de acción, creencias y concepciones relacionadas con la forma de enseñar.

Cabe resaltar que no es suficiente con observar los procesos de enseñanza y aprendizaje que el profesor planea y desarrolla, ya que como menciona Beane (citado por Araya, 2010) es conveniente realizar el análisis de la propia práctica, no individualmente sino en colaboración con pares profesionales, ya que las creencias y concepciones para enseñar pueden pasar desapercibidas a uno mismo o ser validadas por nuestra experiencia. En igual sentido, Porlán, Rivero y Martín del Pozo (1997) plantean que "este tipo de concepciones sólo puede ponerse en evidencia con la ayuda de otras personas (compañeros del equipo de trabajo, formadores de profesores, investigadores, etc.)". Con respecto a estas consideraciones, lo más estratégico sería que las observaciones críticas de las prácticas de aula sean realizadas por pares de otras instituciones educativas o incluso de otras entidades territoriales, en vista de que el mismo tipo de concepciones habitualmente son compartidas entre los compañeros de trabajo. En igual forma, Restrepo (2004, p.54) señala que "la crítica de pares profesionales y la autocrítica de la práctica pedagógica" han sido de gran utilidad para el desarrollo profesional del docente y para la superación de prácticas no influyentes en los procesos de enseñanza y aprendizaje.

Sin embargo, no es suficiente que el docente se observe a sí mismo, que reciba la retroalimentación de la crítica pedagógica de sus pares profesionales o que acceda a la información validada por la comunidad académica. Por esta razón, es indispensable que el docente realice la categorización de sus conocimientos profesionales y sea consistente como influye cada uno de sus componentes en los procesos de enseñanza y aprendizaje. En este sentido, es importante partir de la integración de los conocimientos profesionales del profesor de ciencias (CPPC) para potencializar las habilidades cognitivas y actitudinales de la indagación, por tanto, desarrollar el pensamiento científico de los estudiantes, que como futuros ciudadanos desde la perspectiva de la alfabetización científica tomen decisiones responsables entorno a los avances, limitaciones y riesgos de la ciencia y la tecnología en la sociedad. 
Bio - grafía. Escritos sobre la Biología y su Enseñanza. ISSN 2027-1034

Edición Extraordinaria. p.p. 1238 - 1246

Memorias del IX Encuentro Nacional de Experiencias en Enseñanza de la Biología y la Educación Ambiental. IV Congreso Nacional de Investigación en Enseñanza de la Biología.

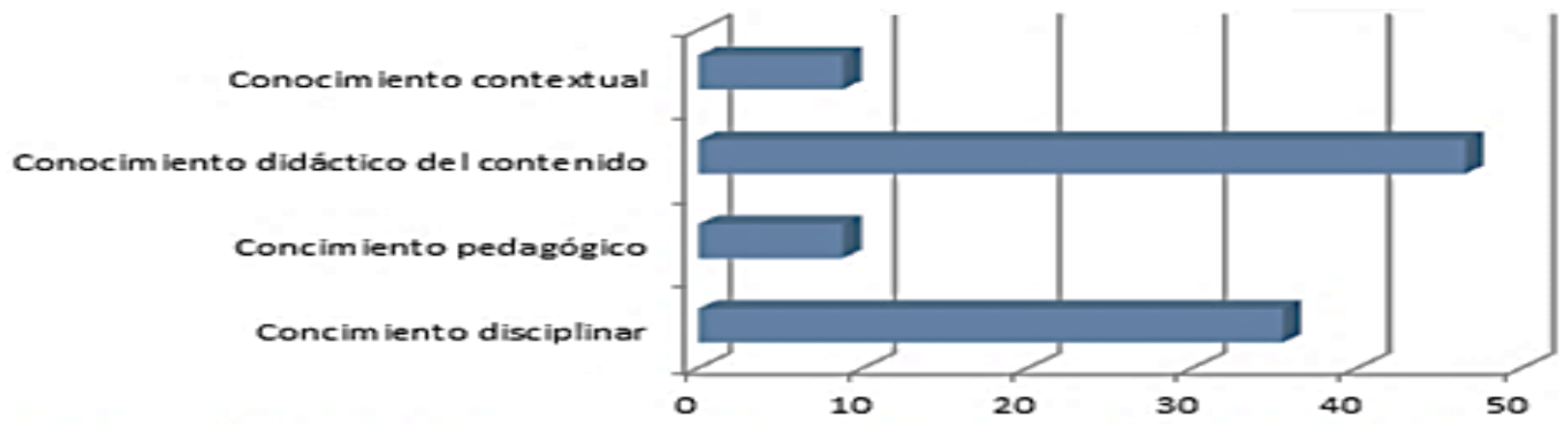

Figura 5: Análisis de los componentes del Conocimiento Profesional del Profesor durante una sesión de clase

En relación con el análisis de los componentes del conocimiento profesional del profesor (figura 5) se obtiene que su relación en las planeaciones y el desarrollo de las clases esté dirigida principalmente hacia la transmisión de contenidos descontextualizados de los intereses y necesidades de la realidad del estudiante. Las debilidades de la clase se evidencian principalmente en la relación del conocimiento pedagógico y contextual. Esta situación es determinante para que la participación de los estudiantes sea escasa, limitada y con poca incidencia para el desarrollo del su pensamiento científico, esto se puede evidenciar en el número de participaciones del profesor - estudiantes y el tiempo de sus intervenciones durante el desarrollo de la clase.

\section{CONCLUSIONES}

La indagación no sólo ofrece posibilidades para potenciar el desarrollo de la alfabetización científica de los estudiantes a partir del fortalecimiento de las habilidades de pensamiento, además le brinda herramientas al docente para que mejore sus prácticas pedagógicas. De acuerdo con las perspectivas expuestas de los procesos de enseñanza y aprendizaje, se debe asumir como docente que los resultados de la evaluación de los estudiantes se constituyen en el reflejo del propio proceso formativo del que enseña y que además, deben ser concebidos como un reto para mejorar la labor del maestro.

Además de la alfabetización científica del estudiante, es conveniente, proponer la "alfabetización académica del docente", tomando como referencia lo que mencionan Radloff y de la Harpe (citados por Carlino, 2003), que consideran la alfabetización académica como los fundamentos teóricos y metodológicos necesarios para participar en las actividades discursivas de las disciplinas mediante la producción y análisis de textos para aprender en la universidad. El docente tiene la oportunidad de aprovechar la información del contexto de enseñanza y aprendizaje para reflexionar, sistematizar, evaluar y elaborar propuestas que fortalezcan el desarrollo del pensamiento científico.

En el caso de la escuela, se visualizan distintas oportunidades para que el profesor, también aprenda a adaptar y mejorar su conocimiento profesional. En este orden de ideas, la "alfabetización académica del docente" es posible cuando reflexiona, sistematiza, planea, 
Bio - grafía. Escritos sobre la Biología y su Enseñanza. ISSN 2027-1034

\section{Edición Extraordinaria. p.p. 1238 - 1246}

Memorias del IX Encuentro Nacional de Experiencias en Enseñanza de la Biología y la Educación Ambiental. IV Congreso Nacional de Investigación en Enseñanza de la Biología.

evalúa y comunica cómo influye y se "articula en su enseñanza la materia que imparte con las características, antecedentes, necesidades e intereses de los alumnos, así como sus propias necesidades, creencias y valores de las enseñanza" (Díaz, 2006, p. 15).

Finalmente, la sociedad requiere de docentes con capacidad autocritica de su labor, que al mismo tiempo se apoyen en los saberes y la capacidad crítica de sus pares profesionales para definir y validar criterios de mejoramiento de los procesos de enseñanza y aprendizaje. En este sentido, un docente reflexivo de su práctica pedagógica no solamente lleva al estudiante a plantear preguntas relacionadas con los eventos de su entorno, a buscar las posibles respuestas y a tomar decisiones como un futuro ciudadano alfabetizado científicamente; también reconstruye continuamente su conocimiento profesional y se proyecta a nivel social como un docente potencializado por las capacidades que le brinda la alfabetización académica.

\section{REFERENCIAS BIBLIOGRÁFICAS}

Araya, V. (2010). ¿Pueden los docentes construir su saber y profesionalidad?, consultado en: 23 de mayo de 1017. Recuperado de: https://goo.gl/LSJyCC.

Blanco, L., Mellado, V. y Ruiz, C. (1995). El conocimiento didáctico del contenido en Ciencias Experimentales y Matemáticas y formación de profesores, consultado en: el 11 de abril de 2017. Recuperado de: https://goo.gl/PYg3dJ.

Carvalho, A. (1997). Cambios didácticos como consecuencia de las innovaciones curriculares. Proyecto Principal de Educación en América Latina y el Caribe, consultado en: 22 de mayo de 1017. Recuperado de: https://goo.gl/Mt2xju.

Carlino, P. (2003). Alfabetización académica: un cambio necesario, algunas alternativas posibles, consultado en: 25 de mayo de 2017. Recuperado de: https://goo.gl/bke4ll. 
Bio - grafía. Escritos sobre la Biología y su Enseñanza. ISSN 2027-1034

Edición Extraordinaria. p.p. 1238 - 1246

Memorias del IX Encuentro Nacional de Experiencias en Enseñanza de la Biología y la Educación Ambiental. IV Congreso Nacional de Investigación en Enseñanza de la Biología.

Daza, S., Quintanilla, M., Arrieta, J. y Monterrosa, E. (2011). La enseñanza de las ciencias en las primeras edades. Vol. 5 [Capítulo 3 . El preguntario de los niños, para cada genuina pregunta existe una respuesta sabia, consultado en: 17 de abril de 2017.Recuperado de: https://goo.gl/84F5lt

Díaz, F. (2006). Enseñanza Situada: Un vínculo entre la escuela y la vida, consultado en: 22 de mayo de 2017. Recuperado de: https://goo.gl/WOrvnO.

Latorre, M. (1992). La reflexión en la formación del profesor, consultado en: 4 de julio de 2017. Recuperado de: https://goo.gl/mBnER7

Márquez, C. (2011). Cuaderno de indagación en el aula y competencia científica. Cómo promover el desarrollo de una competencia científica, Consultado en: 19 de abril de 2017, Recuperado de: $\underline{\text { https://goo.gl/PLdvJH. }}$.

Meinardi, E., González, L., Revel, A. y Victoria, M. (2010). Educar en Ciencias. Buenos Aires, Argentina: Paidós. [Capítulo 1. El sentido de educar en ciencias]

Porlán, R. (1987). El maestro como investigador en el aula. Investigar para conocer, conocer para enseñar, consultado en: 20 de mayo de 2017. Recuperado en: https://goo.gl/S3nnpl.

Porlán, R., Rivero, R. y Martín del Pozo, R. (1997). Conocimiento profesional y epistemología de los profesores I: Teoría, métodos e instrumentos, Consultado en: 22 de mayo de 2017. Recuperado de: https://goo.gl/pvbdRa

Pozo, J y Carretero, M. (1987). Del pensamiento formal a las concepciones espontáneas: ¿Qué cambia en la enseñanza de la ciencia? Infancia y Aprendizaje, Consultado en: 6 de diciembre de 2016, Recuperado: https://goo.gl/vMsp8V

Restrepo, B. (2004). La investigación- acción educativa y la construcción de saber pedagógico, consultado en: 24 de mayo de 2017. Recuperado de: https://goo.gl/N7ACDg

Santelices, L. (1989). Metodología de ciencias naturales para la enseñanza básica. Santiago de chile: Andrés Bello. 